\title{
Optimal Prediction of Carotid Intraplaque Hemorrhage Using Clinical and Lumen Imaging Markers
}

\author{
(D) M.S. McLaughlin, P.J. Hinckley, S.M. Treiman, S.-E. Kim, G.J. Stoddard, D.L. Parker, G.S. Treiman, and J.S. McNally
}

\begin{abstract}
BACKGROUND AND PURPOSE: MR imaging detects intraplaque hemorrhage with high accuracy by using the magnetization-prepared rapid acquisition of gradient echo sequence. Still, MR imaging is not readily available for all patients, and many undergo CTA instead. Our goal was to determine essential clinical and lumen imaging predictors of intraplaque hemorrhage, as indicators of its presence and clues to its pathogenesis.
\end{abstract}

MATERIALS AND METHODS: In this retrospective cross-sectional study, patients undergoing stroke work-up with MR imaging/MRA underwent carotid intraplaque hemorrhage imaging. We analyzed 726 carotid plaques, excluding vessels with non-carotid stroke sources ( $n=420$ ), occlusions $(n=7)$, or near-occlusions $(n=3)$. Potential carotid imaging predictors of intraplaque hemorrhage included percentage diameter and millimeter stenosis, plaque thickness, ulceration, and intraluminal thrombus. Clinical predictors were recorded, and a multivariable logistic regression model was fitted. Backward elimination was used to determine essential intraplaque hemorrhage predictors with a thresholded 2 -sided $P<.10$. Receiver operating characteristic analysis was also performed.

RESULTS: Predictors of carotid intraplaque hemorrhage included plaque thickness ( $O R=2.20, P<.001)$, millimeter stenosis (OR $=0.46$, $P<.001)$, ulceration ( $O R=4.25, P=.020)$, age $(O R=1.11, P=.001)$, and male sex $(O R=3.23, P=.077)$. The final model discriminatory value was excellent (area under the curve $=0.932$ ). This was significantly higher than models using only plaque thickness (area under the curve $=$ 0.881 ), millimeter stenosis (area under the curve $=0.830$ ), or ulceration (area under the curve $=0.715, P<.001$ ).

CONCLUSIONS: Optimal discrimination of carotid intraplaque hemorrhage requires information on plaque thickness, millimeter stenosis, ulceration, age, and male sex. These factors predict intraplaque hemorrhage with high discriminatory power and may provide clues to the pathogenesis of intraplaque hemorrhage. This model could be used to predict the presence of intraplaque hemorrhage when MR imaging is contraindicated.

ABBREVIATIONS: IPH = intraplaque hemorrhage; $\mathrm{AUC}=$ area under the curve

$\mathbf{F}^{\circ}$ or $>20$ years, stenosis has been the primary predictor of stroke risk from carotid disease based on landmark studies including the North American Symptomatic Carotid Endarterectomy Trial, Asymptomatic Carotid Atherosclerosis Study, and European Ca-

Received January 25, 2015; accepted after revision May 3.

From the Department of Radiology (M.S.M., P.J.H., S.M.T., S.-E.K., D.L.P., G.S.T., J.S.M.), Utah Center for Advanced Imaging Research; Department of Orthopedics (G.J.S.), Study Design and Biostatistics Center, and Department of Surgery (G.S.T.), University of Utah, Salt Lake City, Utah; and Department of Surgery (G.S.T.), VA Salt Lake City Health Care System, Salt Lake City, Utah.

This work was supported by the University of Utah Study Design and Biostatistics Center, with funding in part from the National Center for Research Resources and the National Center for Advancing Translational Sciences, National Institutes of Health, through grant 8ULITR000105 (formerly ULIRR025764).

Please address correspondence to J. Scott McNally, MD, PhD, University of Utah, Department of Radiology, 30 N 1900 East, Salt Lake City, UT 84132; e-mail: scott.mcnally@hsc.utah.edu

- Indicates open access to non-subscribers at www.ajnr.org

http://dx.doi.org/10.3174/ajnr.A4454 rotid Surgery Trial. ${ }^{1-3}$ During the past 10 years, advances have been made in MR imaging detection of plaque components, most notably with carotid intraplaque hemorrhage (IPH). Moody et $\mathrm{al}^{4}$ first reported that high MR imaging signal within the carotid wall was shown to detect complex atheromas. Since that time, carotid IPH has been detectable with high accuracy by using heavily T1weighted sequences such as magnetization-prepared rapid acquisition of gradient echo. ${ }^{5}$ MR imaging-detected carotid IPH is an essential indicator of stroke risk and is independent of stenosis. ${ }^{6-9}$ However, MR imaging in general and the MPRAGE sequence specifically may not be available in all clinical settings.

While most reports have correlated IPH with other markers of plaque vulnerability, none have generated a predictive model of IPH based on all available clinical and imaging markers. Many researchers have demonstrated that the likelihood of IPH increases with increasing carotid stenosis. ${ }^{10}$ Others have shown that IPH positively correlates with not only stenosis but also plaque volume or 
thickness. ${ }^{11}$ Recently, CTA-detected ulceration was found to act as a surrogate marker for IPH. ${ }^{12}$ These data suggest that IPH can be predicted, with some degree of accuracy, on the basis of lumen imaging findings. In addition, IPH has been found at a higher prevalence in males and in higher age groups. ${ }^{13}$ With these findings in mind, the goal of this study was to determine a predictive model of IPH by using available imaging and clinical markers.

We began with the hypothesis that lumen imaging and clinical factors could be used to predict the presence of IPH. Doing so may provide clues to the pathogenesis of IPH. Another potential benefit would be to patients in whom MR imaging is contraindicated. To detect IPH, we used an MPRAGE sequence included as part of a clinical protocol beginning in November 2009. Data were gathered prospectively and analyzed in a retrospective cross-sectional study. We included all patients imaged with carotid MRA by using the MPRAGE sequence for 4.5 years. A multivariable logistic regression model was fitted to determine essential imaging and clinical markers of IPH. This cohort of patients was used previously to determine predictors of carotid-source stroke, which were found to include intraluminal thrombus, intraplaque hemorrhage, plaque thickness, and current smoking. ${ }^{8}$

\section{MATERIALS AND METHODS \\ Clinical Study Design}

Institutional review board approval was obtained for this crosssectional study on patients undergoing stroke evaluation with brain MR imaging/carotid MRA from November 2009 to January 2014. The carotid MPRAGE sequence was added to the clinical MRA imaging protocol in November 2009. Patients presenting for stroke work-up were scanned within 1 week of symptom onset. From November 2009 to January 2014, 578 patients underwent brain MR imaging/carotid MRA for acute stroke evaluation with the additional MPRAGE sequence. This resulted in $1156 \mathrm{ca}-$ rotid artery-ipsilateral brain image pairs.

Exclusions were determined after reviewing electronic medical records for non-carotid plaque stroke sources: those outside 2 $\mathrm{cm}$ above and below the carotid bifurcation. We excluded 430 carotid-brain pairs. These included craniocervical dissections ( $n=118)$, atrial fibrillation $(n=94)$, intracardiac/extracardiac shunt $(n=86)$, cardiac thrombus $(n=26)$, recent aortic or mitral valve replacement $(n=16)$, vasculitis $(n=14)$, global hypoxic/ischemic injury $(n=10)$, recent cardiac or neurovascular catheterization $(n=10)$, recent cardiovascular surgery $(n=8)$, dural venous sinus thrombosis $(n=8)$, fibromuscular dysplasia or lupus vasculopathy $(n=8)$, proximal common carotid stenosis $>50 \%(n=6)$, rheumatic heart disease $(n=4)$, brain neoplasm $(n=4)$, endocarditis $(n=2)$, idiopathic hypertrophic subaortic stenosis $(n=2)$, aortic graft complication $(n=2)$, and distal vessel atherosclerosis $(n=2)$. We also excluded occluded carotid arteries $(n=7)$, and extremely high-grade lesions $(n=3)$. We used 726 carotid plaques in the final analysis. Although a few scans showed mild motion artifacts primarily from swallowing, these artifacts were not sufficient to exclude any carotid arteries from interpretation.

\section{MR Imaging/MRA Clinical Protocol}

Images were obtained on 3T and 1.5T MR imaging scanners (Verio and Avanto, Siemens, Erlangen, Germany) with standard head/neck coils. The standard clinical MR imaging/MRA protocol for these patients included brain MR imaging (axial DWI, axial T2-weighted, axial FLAIR, and sagittal T1-weighted images), brain MRA (3D axial TOF), and neck MRA (2D axial TOF, coronal precontrast T1-weighted, and coronal postcontrast arterial and venous phase images). Coronal postcontrast MRA neck images were obtained from the aortic arch through the circle of Willis. Total scan time was approximately 45 minutes, of which the MPRAGE sequence required approximately 5 minutes. In cases in which renal insufficiency precluded intravenous contrast (glomerular filtration rate, $<30 \mathrm{~mL} / \mathrm{min} / 1.73 \mathrm{~m}^{2}$ ), postcontrast MRA images were replaced with $3 \mathrm{D}$ noncontrast TOF with 1-mm section thickness combined with duplex sonography.

\section{Carotid MPRAGE Sequence}

MPRAGE parameters were first optimized at $3 \mathrm{~T}$ and were as follows: $3 \mathrm{D}, \mathrm{TR} / \mathrm{TE} / \mathrm{TI}=6.39 / 2.37 / 370 \mathrm{~ms}$, flip angle $=15^{\circ}, \mathrm{FOV}=$ $130 \times 130 \times 48 \mathrm{~mm}^{3}$, matrix $=256 \times 256 \times 48$, voxel $=0.5 \times$ $0.5 \times 1.0 \mathrm{~mm}^{3}$, fat saturation, time $\sim 5$ minutes. The TI was initially optimized for 3T and transferred to 1.5T. An initial TI of approximately $500 \mathrm{~ms}$ was chosen on the basis of prior computer simulations at $3 \mathrm{~T}$ and was adjusted down to a TI of $370 \mathrm{~ms}$ to maximize contrast between hemorrhage and inflowing blood in human volunteers as described previously. ${ }^{14,15}$ Images were obtained from $20 \mathrm{~mm}$ below to $20 \mathrm{~mm}$ above the carotid bifurcation at $1.0-\mathrm{mm}$ section thickness. To produce $3 \mathrm{D}$ images, we used a secondary phase-encoding gradient in the section-select direction, and measurements for all section-selection phase-encodings were performed with rapid acquisition in each segment.

\section{Carotid Lumen Imaging Markers}

All carotid imaging markers were determined by consensus review of 2 reviewers (M.S.M. and J.S.M.), both blinded to findings on brain MR imaging and clinical covariates. In addition, IPH was determined independently of other carotid imaging markers of stroke risk. Lumen markers included percentage diameter stenosis, millimeter stenosis, maximum plaque thickness, ulceration, and intraluminal thrombus.

Percentage diameter stenosis was determined by using the NASCET criteria on contrast MRA. Briefly, the diameter $(b)$ at the level of maximal stenosis and the diameter $(a)$ of the internal carotid artery distal to the stenosis were used to calculate the percentage diameter stenosis by using the formula $[(a-b) / a] \times$ $100 \%$. Carotid stenosis was measured at the narrowest segment of the carotid plaque $(b)$ on the axial images, perpendicular to the long axis of the vessel on multiplanar reformats by using a submillimeter measurement tool (Fig 1A). The distal ICA diameter (a) was measured beyond the bulb where the walls are parallel and no longer tapering per NASCET criteria. ${ }^{16-18}$ We performed the multivariable regression analysis by using both the NASCET measurement of percentage diameter stenosis $[(a-b) / a] \times 100 \%$ and a millimeter stenosis $(b)$ measurement adapted from the millimeter stenosis method first described on CTA. ${ }^{19}$ To identify near-occlusions, we excluded an ICA from the percentage stenosis calculation if it met the following criteria: visible bulb stenosis, distal ICA diameter of $\leq 3 \mathrm{~mm}$, and distal ICA/distal ECA ratio of $\leq 1.25$. These criteria were further adapted from those used by Bartlett et $\mathrm{al}^{18}$ to recognize

AJNR Am J Neuroradiol 36:2360-66 Dec 2015 www.ajnr.org 2361 


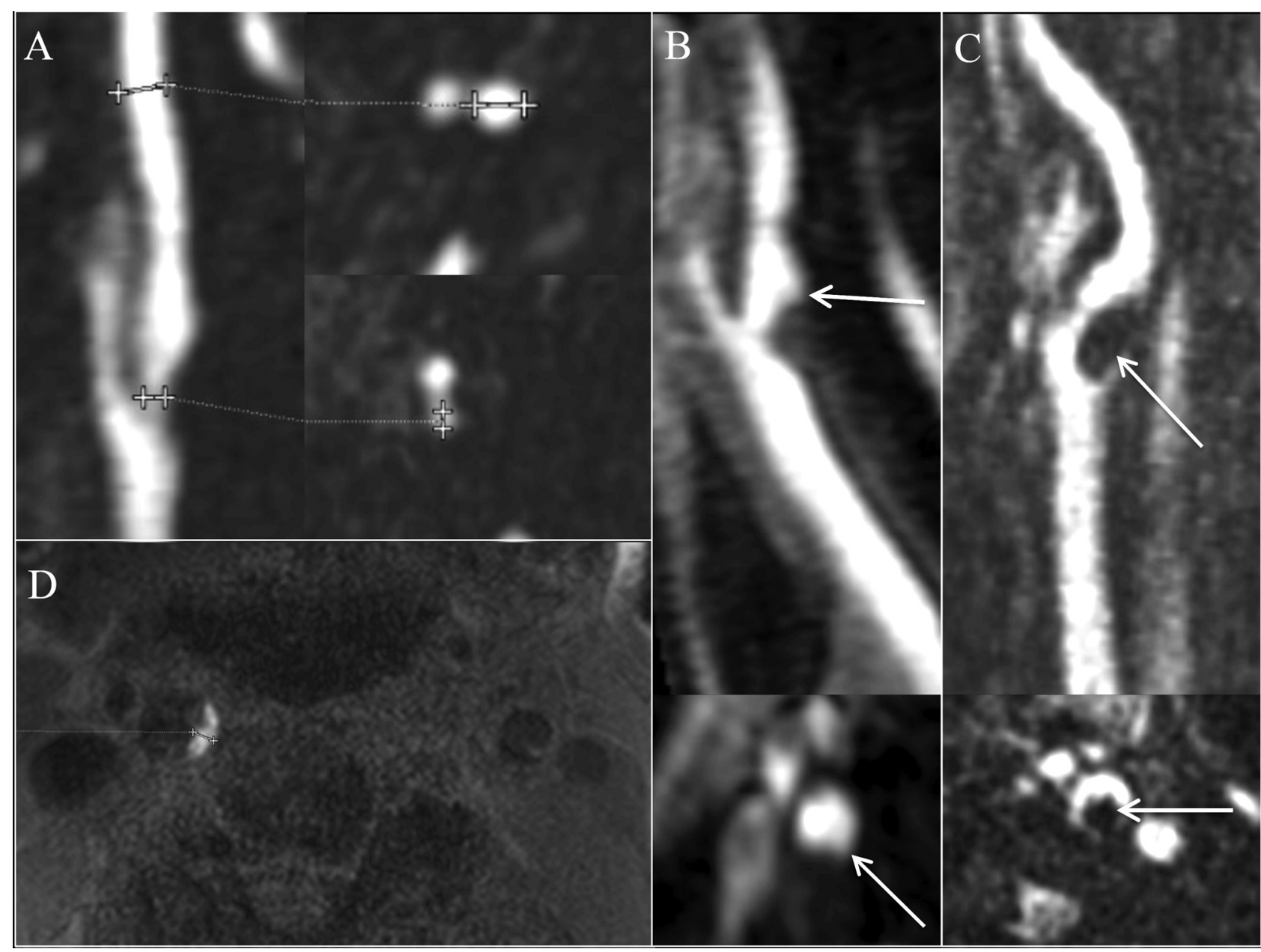

FIG 1. Carotid plaque imaging markers. Stenosis is measured by using percentage diameter stenosis $[(a-b) / a]$ and millimeter stenosis $(b)(A$, cursors). The presence of ulceration is determined on contrast MRA images by using a 2-mm measurement threshold ( $B$, arrow). Intraluminal thrombus is defined as a filling defect on contrast MRA images ( $C$, arrow). IPH is defined by MPRAGE-positive plaque, by using a signal threshold of 2-fold signal intensity over the adjacent sternocleidomastoid muscle ( $D$, right carotid artery is MPRAGE-positive; left side of image). Maximum plaque thickness is measured in the transverse plane on 3D MPRAGE image ( $D$, cursors).

subtle near-occlusions on CTA and originally adapted from standard conventional angiography. ${ }^{18,19}$

The presence of ulceration was determined on contrast MRA images by using a size threshold of $2 \mathrm{~mm}$ as previously described with CTA (Fig $1 B){ }^{12}$ Intraluminal thrombus was defined by an intraluminal filling defect on MRA axial reformats, previously described on CTA (Fig 1C). ${ }^{20}$ In arteries from patients with renal failure (glomerular filtration rate, $<30 \mathrm{~mL} / \mathrm{min} / 1.73 \mathrm{~m}^{2} ; 68 / 726$; or $9.4 \%$ of carotidbrain image pairs), the above imaging markers were determined from 3D noncontrast TOF with 1-mm section thickness combined with duplex sonography. Maximum plaque thickness was measured in the transverse plane on MPRAGE images (Fig 1D). IPH was defined quantitatively as MPRAGE-positive plaque, with at least 1 voxel demonstrating at least a 2 -fold higher signal intensity relative to the adjacent sternocleidomastoid muscle as previously described (Fig $1 D$; right carotid is MPRAGE-positive; left side of image). ${ }^{6}$

\section{Statistical Analysis}

A mixed-effects multivariable logistic regression model was used. This model accounted for 2 vessels per patient. The multivariable logistic regression model was fitted for the outcome of carotid $\mathrm{IPH}$, with carotid imaging predictors including percentage diam- eter stenosis, millimeter stenosis, maximum plaque thickness, ulceration, and intraluminal thrombus. Clinical covariates included age, male sex, diabetes, hypertension, hyperlipidemia, and body mass index. Cardiovascular medication confounders included antihypertension, antiplatelet, anticoagulation, and statin medication classes. In addition, magnet strength (3T or $1.5 \mathrm{~T}$ ) was included as a potential confounder in the logistic regression model. A backward-elimination method was used to determine the final model, in which all remaining predictors had a $P<.10$. Odds ratios and $P$ values were reported for each factor alone and for the factors found to be significant from the backward elimination. Receiver operating characteristic comparison analysis was performed to determine the discriminatory value of the final model compared with the following: 1) a model using only plaque thickness, 2) a model using only percentage stenosis as a continuous variable, and 3) a model by using only plaque ulceration. All statistical analyses were performed with STATA, Version 13.1 (StataCorp, College Station, Texas).

\section{RESULTS}

\section{Patient Demographics}

Patient demographics are listed by vessel and depicted in Table 1. 


\section{Carotid Plaque Markers and Stroke Imaging}

The carotid imaging features used in this study included the outcome, IPH, and predictors, including percentage diameter stenosis, millimeter stenosis, maximum plaque thickness, ulceration, and intraluminal thrombus as described in the "Materials and Methods" section (Fig 1).

\section{Multivariable Logistic Regression}

Intraplaque hemorrhage predictors are depicted in Table 2,

Table 1: Patient demographics

\begin{tabular}{lc}
\hline \multicolumn{1}{c}{ Carotid Stroke Predictor } & $\begin{array}{c}\text { Demographics } \\
\text { by Vessel }\end{array}$ \\
\hline Male sex (No./total No.) (\%) & $387 / 726(53.3)$ \\
Age (yr) (mean) (SD) & $64.2(15.6)$ \\
BMI (mean) (SD) (kg/m²) & $28.4(6.4)$ \\
Smoking (No./total No.) (\%) & \\
$\quad$ Current smoker & $138 / 726(19.0)$ \\
Prior smoker & $158 / 726(21.8)$ \\
Never smoked & $430 / 726(59.2)$ \\
Hypertension (No./total No.) (\%) & $499 / 726(68.7)$ \\
Hyperlipidemia (No./total No.) (\%) & $358 / 726(49.3)$ \\
Diabetes (No./total No.) (\%) & $227 / 726(31.3)$ \\
Cardiovascular medications & \\
Antihypertension (No./total No.) (\%) & $412 / 726(56.8)$ \\
Statins (No./total No.) (\%) & $316 / 726(43.5)$ \\
Antiplatelet (No./total No.) (\%) & $294 / 726(40.5)$ \\
Anticoagulation (No./total No.) (\%) & $74 / 726(10.2)$ \\
Carotid plaque imaging markers & \\
Stenosis (mean) (SD) (\%) & $12.2(23.1)$ \\
$\quad$ Mild stenosis (0\%-49\%) (No./total No.) (\%) & $647 / 726(89.1)$ \\
$\quad$ Moderate stenosis (50\%-69\%) (No./total No.) (\%) & $45 / 726(6.2)$ \\
$\quad$ Severe stenosis (70\%-99\%) (No./total No.) (\%) & $34 / 726(4.7)$ \\
Stenosis (mean) (SD) (mm) & $4.1(1.2)$ \\
Maximum plaque thickness (mean) (SD) (mm) & $3.0(1.6)$ \\
Ulceration (No./total No.) (\%) & $96 / 726(13.2)$ \\
Intraluminal thrombus (No./total No.) (\%) & $19 / 726(2.6)$ \\
Intraplaque hemorrhage (No./total No.) (\%) & $65 / 726(9.0)$ \\
Magnet strength = 3T (No./total No.) (\%) & $58 / 726(8.0)$ \\
\hline Note-BMindicas body mass index. &
\end{tabular}

with odds ratios adjusted by using multivariable logistic regression.

\section{Final Multivariable Logistic Regression Model}

The final model for predictors of carotid IPH is depicted in Table 3. After backward elimination with a threshold of $P<.10$, the remaining significant factors predicting carotid IPH included maximum plaque thickness $(\mathrm{OR}=2.20 ; P<.001 ; 95 \%$ confidence interval, 1.50-3.22), millimeter stenosis $(\mathrm{OR}=0.46$; $P<.001 ; 95 \%$ CI, 0.30-0.71), ulceration $(\mathrm{OR}=4.25 ; P=.020 ; 95 \% \mathrm{CI}$, $1.25-14.4)$, age $(\mathrm{OR}=1.11 ; P=.001$; $95 \% \mathrm{CI}, 1.05,1.18)$, and male sex $(\mathrm{OR}=$ $3.23 ; P=.077 ; 95 \% \mathrm{CI}, 0.88,11.9)$. Note that millimeter stenosis is a measure of the lumen diameter $(b)$ at the level of stenosis described in the "Materials and Methods" section. Thus, carotid plaque with severe stenosis (small $b$ ) is associated with IPH, and carotid plaque without stenosis (large $b$ ) is not associated with IPH. This difference accounts for the seemingly counterintuitive OR of 0.46 in the final model. In addition, percentage diameter stenosis measured by NASCET is not in the final model due to the nonsignificant $P>.10$ during backward elimination. If, alternatively, millimeter stenosis was not measured, then percentage stenosis would meet the $P<.10$ criteria (full model: $\mathrm{OR}=23.7$; $P=.003 ; 95 \% \mathrm{CI}, 3.00-187.2$; final model: $\mathrm{OR}=31.0 ; P=.001 ; 95 \% \mathrm{CI}$, 3.89-246.2). However, because we measured both percentage and millimeter

Note:-BMl indicates body mass index.

Table 2: Multivariable logistic regression

\begin{tabular}{|c|c|c|c|c|c|c|}
\hline \multirow{2}{*}{$\begin{array}{c}\text { Carotid-IPH Predictor } \\
\text { Cardiovascular risk factors }\end{array}$} & \multirow[t]{2}{*}{$\mathrm{IPH}+(n=65)$} & \multirow[t]{2}{*}{ IPH- $(n=661)$} & \multirow[t]{2}{*}{ OR } & \multirow[t]{2}{*}{$P$ Value } & \multicolumn{2}{|c|}{$95 \% \mathrm{Cl}$} \\
\hline & & & & & & \\
\hline Male sex (No./total No.) (\%) & $55 / 65(70.0)$ & $332 / 661(50.2)$ & 3.05 & .104 & 0.79 & 11.7 \\
\hline Age (yr) (mean) (SD) & $76.0(9.7)$ & $63.1(15.6)$ & 1.11 & .003 & 1.04 & 1.19 \\
\hline BMI (yr) (mean) $\left(\mathrm{kg} / \mathrm{m}^{2}\right)$ & $26.8(4.0)$ & $28.5(6.6)$ & 0.94 & .325 & 0.84 & 1.06 \\
\hline \multicolumn{7}{|l|}{ Smoking (No./total No.) (\%) } \\
\hline Current smoker & $12 / 65(18.5)$ & $126 / 661(19.1)$ & 1.52 & .620 & 0.29 & 7.88 \\
\hline Prior smoker & $24 / 65(36.9)$ & $134 / 661(20.3)$ & 1.74 & .399 & 0.48 & 6.36 \\
\hline Hypertension (No./total No.) (\%) & $46 / 65(70.8)$ & $453 / 661(68.5)$ & 0.30 & .126 & 0.06 & 1.41 \\
\hline Hyperlipidemia (No./total No.) (\%) & $46 / 65(70.8)$ & $312 / 661(47.2)$ & 1.08 & .895 & 0.33 & 3.62 \\
\hline Diabetes (No./total No.) (\%) & $23 / 65(35.4)$ & $204 / 661(30.9)$ & 1.04 & .948 & 0.33 & 3.30 \\
\hline \multicolumn{7}{|l|}{ Cardiovascular medications (No./total No.) (\%) } \\
\hline Antihypertension & $43 / 65(66.2)$ & $369 / 661(55.8)$ & 2.70 & .170 & 0.65 & 11.1 \\
\hline Statin & $42 / 65(64.6)$ & $274 / 661(41.5)$ & 1.24 & .749 & 0.33 & 4.70 \\
\hline Antiplatelet & $38 / 65(58.5)$ & $256 / 661(38.7)$ & 1.07 & .922 & 0.29 & 3.88 \\
\hline Anticoagulation & $7 / 65(10.8)$ & $67 / 661(10.1)$ & 0.63 & .628 & 0.10 & 4.00 \\
\hline \multicolumn{7}{|l|}{ Carotid plaque imaging markers } \\
\hline Stenosis (mean) (SD) (\%) & $46.5(30.1)$ & $8.9(19.3)$ & .09 & .409 & 0.0003 & 25.7 \\
\hline Stenosis (mean) (SD) (mm) & $2.5(1.5)$ & $4.3(1.0)$ & 0.31 & .052 & 0.09 & 1.01 \\
\hline Maximum plaque thickness (mean) (SD) (mm) & $5.4(1.9)$ & $2.8(1.4)$ & 2.26 & $<.001$ & 0.09 & 1.01 \\
\hline Ulceration (No./total No.) (\%) & $34 / 65(52.3)$ & 62/661 (9.4) & 4.36 & .017 & 1.30 & 14.7 \\
\hline Intraluminal thrombus (No./total No.) (\%) & $5 / 65(7.7)$ & $14 / 661(2.1)$ & 0.49 & .496 & 0.06 & 3.85 \\
\hline Magnet strength $=3 \mathrm{~T}$ (No./total No.) (\%) & $16 / 65(24.6)$ & $42 / 661(6.4)$ & 1.63 & .544 & 0.34 & 7.82 \\
\hline
\end{tabular}

Note:-BMI indicates body mass index. 
Table 3: Final model

\begin{tabular}{llrrr}
\hline \multicolumn{1}{c}{ Carotid IPH predictor } & OR & $\boldsymbol{P}$ Value & \multicolumn{2}{c}{$\mathbf{9 5 \%} \mathbf{C I}$} \\
\hline Ulceration & 4.25 & .020 & 1.25 & 14.4 \\
Male sex & 3.23 & .077 & 0.88 & 11.9 \\
Maximum plaque thickness & 2.20 & $<.001$ & 1.50 & 3.22 \\
Age & 1.11 & .001 & 1.05 & 1.18 \\
Stenosis (mm) & 0.46 & $<.001$ & 0.30 & 0.71 \\
\hline
\end{tabular}

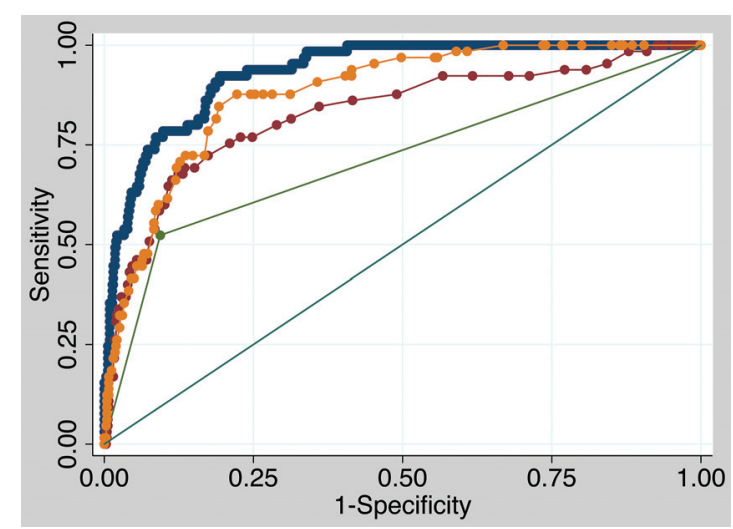

FIG 2. Receiver operating characteristic comparison analysis. The final model IPH discriminatory value is excellent (blue, AUC $=0.932$ ). The final model discriminatory value (blue) is significantly higher than a model with maximum plaque thickness only (yellow, $\mathrm{AUC}=0.881$, $P<.001$ ), a model with millimeter stenosis only (red, AUC $=0.830$, $P<.001$ ), and a model with ulceration only (green, AUC $=0.715, P<.001$ ).

stenosis and millimeter stenosis was a better predictor of IPH, we have kept it in the final model.

\section{Receiver Operating Characteristic Comparison Analysis}

Receiver operating characteristic comparison analysis is shown in Fig 2. The final model discriminatory value was excellent (area under the curve $[$ AUC $]=0.932$ ) and was significantly higher than models using only plaque thickness (AUC $=0.881$ ), only millimeter stenosis $(\mathrm{AUC}=0.830)$, or only ulceration $(\mathrm{AUC}=0.715$, $P<.001$.

\section{DISCUSSION}

Along with other clinical and imaging factors, carotid IPH allows optimal prediction of carotid sources of stroke. ${ }^{8}$ Currently, optimal medical treatment for carotid IPH is unknown. It is, therefore, essential to determine predictors of IPH. These predictors could be used as surrogate markers to calculate the likelihood for IPH when MR imaging is not available or is contraindicated. In addition, these predisposing factors may serve as clues to the pathogenesis of IPH.

Lumen imaging is far more often used in the work-up of carotid-source stroke. CTA is also used more frequently than MRA, by as much as 4 times at our institutions. Lumen imaging of stenosis alone provides poor prediction of IPH. ${ }^{21}$ This study demonstrates that the presence of lumen markers, such as maximum plaque thickness, millimeter stenosis, and ulceration combined with the patient's age and whether the patient is male, predict carotid IPH with high discriminatory power.

These results indicate that plaque ulceration is strongly predictive of IPH. This finding may be because both ulceration and IPH are markers of unstable plaque and frequently coexist. Alternatively, IPH may predispose to endothelial dysfunction, erosion, and eventual ulceration through proinflammatory effects of iron on reactive oxygen species formation. ${ }^{22}$ Plaque ulceration has been previously suggested as a surrogate marker of carotid IPH. ${ }^{12}$ Our research argues that while ulceration is an essential predictor of IPH, it cannot fully act as a surrogate for IPH without determining the other clinical and imaging factors in the regression analysis. In the assessment of carotid stroke risk, the presence of ulceration alone could prompt further evaluation with MR imaging to assess IPH.

Our study also shows that maximum plaque thickness is an essential predictor of IPH. This suggests that larger plaques are inherently more unstable and prone to hemorrhage, potentially due to a larger lipid-rich core and/or a higher number or more permeable plaque neovessels. Delayed contrast imaging may allow better detection of lipid-rich cores, and dynamic contrastenhanced MR imaging may better characterize microvascularity predisposing to IPH. ${ }^{23,24}$

In addition to plaque thickness, millimeter stenosis was a significant indicator of IPH. Most interesting, millimeter stenosis was a better predictor than percentage diameter stenosis, and when both were used, percentage stenosis was eliminated from the final model due to its failure to achieve significance. In support of this, millimeter stenosis has been shown to be a reliable measurement with low interobserver variability. ${ }^{19}$ The single measurement of millimeter stenosis is without the inherent variability of NASCET ratios with 2 measurements and variable distal ICA caliber within and between patients.

It is unclear why millimeter stenosis adds further value to plaque thickness in the prediction of IPH. One possibility is that higher degrees of stenosis are associated with further impaired flow dynamics and oscillatory shear stress. In apolipoprotein E mice on a Western diet and treated with angiotensin II and carotid ligation, IPH develops in areas of stenosis and low wall shear stress. ${ }^{25}$ This is likely related to altered endothelial cell mechanotransduction, because low mean shear stress and oscillatory shear stress lead to endothelial reactive oxygen species formation in cell culture models. ${ }^{26-29}$ The shear stress environment at branch points and stenotic vessels could act to sustain IPH through oxidative stress, chronic plaque inflammation, and sustained neovessel permeability.

Of the clinical factors assessed in this study, only patient age and male sex were found to significantly increase the risk of IPH. Both of these factors have been associated with IPH in recent research. ${ }^{13}$ Atherosclerosis has long been known to be an agerelated phenomenon. Arterial plaques form preferentially at branch points and may be fundamental to the process of aging, having been found in ancient mummies from at least 4 different cultures. ${ }^{30}$ Aging may lead to atherosclerosis via increased levels of oxidative stress, DNA damage, mitochondrial dysfunction, and altered balance of cell proliferation and apoptosis. ${ }^{31}$

While the correlation with age is not surprising, what specific role male sex plays in the development of IPH remains to be seen. It has long been known that atherosclerosis incidence is lower in women compared with men, but this increases after menopause, suggesting an atheroprotective effect of estrogen. ${ }^{32}$ However, randomized controlled clinical trials have found no benefit to estrogen therapy in cardiovascular disease. ${ }^{33}$ Androgens may also benefit male patients through direct action on the vasculature or 
through a more favorable lipid profile, though the effect of androgen therapy after andropause is unknown. ${ }^{34}$ There may also be sex differences accounting for platelet activation, coagulation, and endothelial cell function that may contribute to plaque inflammation and IPH. ${ }^{35}$

In conclusion, this study identifies lumen markers and clinical factors that can predict IPH with a high discriminatory power. These predictors provide clues to the pathogenesis of IPH. In addition, when MR imaging is not available or contraindicated, these markers may allow clinicians to estimate the likelihood of IPH being present. Identifying patients who are likely to be negative for IPH can prevent unneeded surgeries or interventions. Furthermore, prescreening patients before they undergo MRA can significantly improve the positive or negative predictive value of T1-weighted sequences in identifying IPH. This will be important in recruiting patients for future studies aimed at determining optimal IPH treatment.

\section{CONCLUSIONS}

Optimal prediction of carotid IPH is achieved by using information on maximum plaque thickness, millimeter stenosis, ulceration, patient age, and male sex. These factors can be used to determine IPH with a high discriminatory power and may provide clues to the pathogenesis of IPH. Together, they may also be used to determine whether IPH is present in patients in whom MR imaging is contraindicated.

Disclosures: Michael S. McLaughlin—RELATED: Grant: National Center for Research Resources and the National Center for Advancing Translational Sciences, National Institutes of Health,* Comments: grant 8ULITR000105 (formerly ULIRR025764). J. Scott McNally—RELATED: Grant: Radiological Society of North America Research Scholar Grant 2014-2016. * Money paid to the institution.

\section{REFERENCES}

1. North American Symptomatic Carotid Endarterectomy Trial Collaborators. Beneficial effect of carotid endarterectomy in symptomatic patients with high-grade carotid stenosis. N Engl J Med 1991;325: 445-53 CrossRef Medline

2. Endarterectomy for asymptomatic carotid artery stenosis: Executive Committee for the Asymptomatic Carotid Atherosclerosis Study. JAMA 1995;273:1421-28 CrossRef Medline

3. Randomised trial of endarterectomy for recently symptomatic carotid stenosis: final results of the MRC European Carotid Surgery Trial (ECST). Lancet 1998;351:1379-87 CrossRef Medline

4. Moody AR, Murphy RE, Morgan PS, et al. Characterization of complicated carotid plaque with magnetic resonance direct thrombus imaging in patients with cerebral ischemia. Circulation 2003;107: 3047-52 CrossRef Medline

5. Ota H, Yarnykh VL, Ferguson MS, et al. Carotid intraplaque hemorrhage imaging at 3.0-T MR imaging: comparison of the diagnostic performance of three T1-weighted sequences. Radiology 2010;254: 551-63 CrossRef Medline

6. Yamada N, Higashi M, Otsubo R, et al. Association between signal hyperintensity on T1-weighted MR imaging of carotid plaques and ipsilateral ischemic events. AJNR Am J Neuroradiol 2007;28:287-92 Medline

7. Takaya N, Yuan C, Chu B, et al. Association between carotid plaque characteristics and subsequent ischemic cerebrovascular events: a prospective assessment with MRI-initial results. Stroke 2006;37: 818-23 CrossRef Medline

8. McNally JS, McLaughlin MS, Hinckley PJ, et al. Intraluminal thrombus, intraplaque hemorrhage, plaque thickness, and current smok- ing optimally predict carotid stroke. Stroke 2015;46:84-90 CrossRef Medline

9. McNally JS, Kim SE, Yoon HC, et al. Carotid magnetization-prepared rapid acquisition with gradient-echo signal is associated with acute territorial cerebral ischemic events detected by diffusionweighted MRI. Circ Cardiovasc Imaging 2012;5:376-82 CrossRef Medline

10. Sun J, Song Y, Chen $\mathrm{H}$, et al. Adventitial perfusion and intraplaque hemorrhage: a dynamic contrast-enhanced MRI study in the carotid artery. Stroke 2013;44:1031-36 CrossRef Medline

11. Zhao X, Underhill HR, Zhao Q, et al. Discriminating carotid atherosclerotic lesion severity by luminal stenosis and plaque burden: a comparison utilizing high-resolution magnetic resonance imaging at 3.0 Tesla. Stroke 2011;42:347-53 CrossRef Medline

12. U-King-Im JM, Fox AJ, Aviv RI, et al. Characterization of carotid plaque hemorrhage: a CT angiography and MR intraplaque hemorrhage study. Stroke 2010;41:1623-29 CrossRef Medline

13. Zhao XQ, Hatsukami TS, Hippe DS, et al; AIM-HIGH Carotid MRI Sub-study Investigators. Clinical factors associated with high-risk carotid plaque features as assessed by magnetic resonance imaging in patients with established vascular disease (from the AIM-HIGH Study). Am J Cardiol 2014;114:1412-19 CrossRef Medline

14. Zhu DC, Ferguson MS, DeMarco JK. An optimized 3D inversion recovery prepared fast spoiled gradient recalled sequence for carotid plaque hemorrhage imaging at 3.0 T. Magn Reson Imaging 2008;26:1360-66 CrossRef Medline

15. Hadley JR, Roberts JA, Goodrich KC, et al. Relative RF coil performance in carotid imaging. Magn Reson Imaging 2005;23:629-39 CrossRef Medline

16. North American Symptomatic Carotid Endarterectomy Trial: methods, patient characteristics, and progress. Stroke 1991;22: 711-20 CrossRef Medline

17. Fox AJ. How to measure carotid stenosis. Radiology 1993;186: 316-18 CrossRef Medline

18. Bartlett ES, Walters TD, Symons SP, et al. Quantification of carotid stenosis on CT angiography. AJNR Am J Neuroradiol 2006;27:13-19 Medline

19. Fox AJ, Eliasziw M, Rothwell PM, et al. Identification, prognosis, and management of patients with carotid artery near occlusion. AJNR Am J Neuroradiol 2005;26:2086-94 Medline

20. Menon BK, Singh J, Al-Khataami A, et al; Calgary CTA Study Group. The donut sign on CT angiography: an indicator of reversible intraluminal carotid thrombus? Neuroradiology 2010;52:1055-56 CrossRef Medline

21. Anzidei M, Napoli A, Zaccagna F, et al. Diagnostic accuracy of colour Doppler ultrasonography, CT angiography and blood-pool-enhanced MR angiography in assessing carotid stenosis: a comparative study with DSA in 170 patients. Radiol Med 2012;117:54-71 CrossRef Medline

22. Buttari B, Profumo E, Businaro R, et al. Oxidized haemoglobindriven endothelial dysfunction and immune cell activation: novel therapeutic targets for atherosclerosis. Curr Med Chem 2013;20: 4806-14 CrossRef Medline

23. Kerwin WS, O’Brien KD, Ferguson MS, et al. Inflammation in carotid atherosclerotic plaque: a dynamic contrast-enhanced MR imaging study. Radiology 2006;241:459-68 CrossRef Medline

24. Mendes J, Parker DL, McNally S, et al. Three-dimensional dynamic contrast enhanced imaging of the carotid artery with direct arterial input function measurement. Magn Reson Med 2014;72:816-22 CrossRef Medline

25. Cheng C, Tempel D, van Haperen R, et al. Atherosclerotic lesion size and vulnerability are determined by patterns of fluid shear stress. Circulation 2006;113:2744-53 CrossRef Medline

26. McNally JS, Davis ME, Giddens DP, et al. Role of xanthine oxidoreductase and $\mathrm{NAD}(\mathrm{P}) \mathrm{H}$ oxidase in endothelial superoxide production in response to oscillatory shear stress. Am J Physiol Heart Circ Physiol 2003;285:H2290-97 CrossRef Medline 
27. Davies PF, Polacek DC, Shi C, et al. The convergence of haemodynamics, genomics, and endothelial structure in studies of the focal origin of atherosclerosis. Biorheology 2002;39:299-306 Medline

28. Pedersen EM, Oyre S, Agerbaek M, et al. Distribution of early atherosclerotic lesions in the human abdominal aorta correlates with wall shear stresses measured in vivo. Eur J Vasc Endovasc Surg 1999; 18:328-33 CrossRef Medline

29. Wentzel JJ, Kloet J, Andhyiswara I, et al. Shear-stress and wall-stress regulation of vascular remodeling after balloon angioplasty: effect of matrix metalloproteinase inhibition. Circulation 2001;104:91-96 CrossRef Medline

30. Clarke EM, Thompson RC, Allam AH, et al. Is atherosclerosis fundamental to human aging? Lessons from ancient mummies. J Cardiol 2014;63:329-34 CrossRef Medline
31. Sobenin IA, Zhelankin AV, Sinyov VV, et al. Mitochondrial aging: focus on mitochondrial DNA damage in atherosclerosis-a minireview. Gerontology 2015;61:343-49 CrossRef Medline

32. Akishita M, Yu J. Hormonal effects on blood vessels. Hypertens Res 2012;35:363-69 CrossRef Medline

33. Howard BV, Rossouw JE. Estrogens and cardiovascular disease risk revisited: the Women's Health Initiative. Curr Opin Lipidol 2013;24: 493-99 CrossRef Medline

34. Traish AM, Kypreos KE. Testosterone and cardiovascular disease: an old idea with modern clinical implications. Atherosclerosis 2011; 214:244-48 CrossRef Medline

35. Roy-O’Reilly M, McCullough LD. Sex differences in stroke: the contribution of coagulation. Exp Neurol 2014;259:16-27 CrossRef Medline 